\title{
Método de diagnóstico da síndrome da apnéia obstrutiva do sono em grau leve através de aprendizado de máquina
}

\author{
Brenda I. C. F. Soares ${ }^{1}$ \\ UFMA, São Luís, MA \\ Luis Fillype da Silva ${ }^{2}$ \\ UFMA, São Luís, MA \\ Allan K. Barros ${ }^{3}$ \\ UFMA, São Luís, MA \\ Jonathan A. Queiroz ${ }^{4}$ \\ UFMA, São Luís, MA
}

\begin{abstract}
Resumo. A Síndrome da Apnéia Obstrutiva do Sono (SAOS) é caracterizada pela fragmentação e hipoxia repetitiva durante o sono, quando não diagnosticada e tratada da forma adequada se torna causa de complicações graves associadas a doenças cardiovasculares. O diagnóstico dessa síndrome requer um estudo clínico detalhado, denominado polissonografia, composto por uma série de exames, dificultando o processo de diagnóstico da SAOS. O exame de polissonografia é considerado padrão para diagnóstico e avaliação dos distúrbios respiratórios do sono, como o próprio nome sugere, este exame reúne diversos testes que realizam análise da atividade cerebral (EEG), cardíaca (ECG), ocular (EOG) e muscular (EMG). Devido à complexidade na realização da polissonografia, o presente trabalho objetiva classificar e diagnosticar dois grupos de indivíduos, saudáveis e com SAOS em grau leve, a partir dos sinais de ECG aplicados em rede neural MLP juntamente à análise de componentes principais (PCA). Ao utilizar a metodologia de extração de características, adaptada para diagnóstico da apnéia obstrutiva do sono, os resultados foram amostrados em duas e três dimensões com classificação de $95 \%$ de acurácia.
\end{abstract}

Palavras-chave. Apnéia, Eletrocardiograma, Poslissonografia, Diagnóstico.

\section{Introdução}

A Síndrome da Apnéia Obstrutiva do Sono (SAOS) se caracteriza pelo comprometimento total (apnéia) ou parcial (hipopnéia) das vias aéreas, por período maior ou igual a 10 segundos, durante o sono [6] [1]. Indivíduos portadores da SAOS apresentam consequências comuns para essa condição, tais como despertares frequentes, ronco, sono agitado, sonolência diurna e problemas cardiovasculares, como hipertensão arterial e arritmia cardíaca [3].

A gravidade da SAOS é determinada a partir do Índice de Apnéia-Hipopnéia (IAH), que representa a quantidade da ocorrência dos sintomas da SAOS por hora de sono. O IAH é dividido em três grupos, grau leve para índice maior que 5 e menor que 15 vezes; Grau moderado quando há valores maiores que 15 e menores que 30; e grau acentuado com IAH maior que grau 30 [10].

\footnotetext{
${ }^{1}$ brenda $_{i}$ rla@hotmail.com

2 silvaluis@outlook.com

${ }^{3}$ queirozjth@gmail.com

4 allan@ufma.br
} 
O diagnóstico da SAOS é realizado por meio da análise do quadro clínico do paciente, que engloba aplicação de questionários, escalas de avaliação do sono e exame quantitativo específico, a polissonografia [4] [8]. O estudo polissonográfico é considerado padrão para diagnóstico e avaliação dos distúrbios respiratórios do sono.

O exame de polissonografia, como o próprio nome sugere, reúne diversos testes que realizam análise da atividade cerebral (Eletroencefalograma - EEG), cardíaca (Eletrocardiograma - ECG), muscular (Eletromiografia - EMG) e ocular (Eletro-oculograma - EOG). Devido a grande quantidade de equipamentos e atendimentos pessoais necessários, o estudo polissonográfico é caracterizado como um exame muito complexo, propõe-se assim a avaliação dos dados de um dos exames presentes na polissonografia, o eletrocardiograma (ECG) em conjunto com técnicas de aprendizado de máquinas, para realizar diagnóstico da SAOS de forma mais viável.

Em Queiroz et. al [9] é realizada a análise dos batimentos cardíacos através de métodos estatísticos para extração de características das bases de dados de ECG. A partir de [9] e da metodologia de classificação em diferentes grupos adotada em [12], o presente trabalho se propõe realizar a separação dos sinais de ECG em dois grupos (SAOS e Saudável), ao utilizar algoritmo de aprendizado de máquinas aliado à análise de componentes principais (PCA) para diagnosticar indivíduos portadores da SAOS em grau leve.

\section{Metodologia}

A metodologia adotada no presente trabalho é baseada na metodologia apresentada em [12] e [9]. Foram utilizados dois grupos distintos como pacientes para realização do exame de ECG, pessoas diagnosticadas com apnéia obstrutiva do sono e pessoas saudáveis. Os sinais foram préprocessados para criação de matrizes contendo os batimentos cardíacos, obtidos a partir dos eletrocardiogramas realizados, dessa forma os valores da mediana, curtose e assimetria foram calculados individualmente para cada base de dados.

\subsection{Base de dados}

As bases de dados utilizadas foram os sinais de eletrocardiograma dos indivíduos saudáveis (MIT-BIH Rhythm Sinus Normal) e dos indivíduos diagnosticados com SAOS (Apnea-ECG Database), ambos presentes em [2]. A base de dados para grupo de indivíduos saudáveis contém 8 gravações, enquanto a base de dados para grupo com SAOS contém 20 gravações, sendo essas classificadas como apnéia em grau leve.

\subsection{Segmentação e pré-processamento dos ciclos cardíacos}

Os batimentos cardíacos de cada indivíduo foram retirados a partir das gravações dos sinais de ECG, amostrados em uma frequência de $128 \mathrm{~Hz}$ para ambos os grupos. Como proposto em Queiroz et. al [9], cada sinal de ECG foi segmentado a fim de se obter os batimentos de cada indivíduo. Em seguida, foi realizado o procedimento presente na equação (1), onde a média dos batimentos foi subtraída do sinal e esse resultado foi dividido pela entropia de Shannon:

$$
b_{n, m}=\frac{b_{n, m}-\frac{1}{N} \sum_{1}^{N} b_{n, m}}{-\sum_{1}^{n} b_{n, m}\left(\log _{2} \frac{1}{N}\right)}
$$

sendo $\mathrm{n}$ representa o número de batimentos, e m representa o número total de coluna de todos os batimentos. 
Os batimentos cardíacos $b_{n, m}$ de cada indivíduo foram concatenados em matrizes. Indivíduos do grupo saudável possuem seus batimentos agrupados na matriz A, enquanto os indivíduos diagnosticados com SAOS estão na matriz B, como mostrada na equação (2):

$$
B=\left[\begin{array}{cccc}
b_{1,1} & b_{1,2} & \ldots & b_{1, n} \\
b_{2,1} & b_{2,2} & \ldots & b_{2, n} \\
\vdots & \vdots & \ddots & \vdots \\
b_{m, 1} & b_{m, 2} & \ldots & b_{m, n}
\end{array}\right]
$$

\subsection{PCA - Principal component analysis}

A partir das matrizes de batimentos cardíacos obtidas através da equação (2), foi necessário realizar uma redução de dimensionalidade das informações contidas nessas matrizes. Dessa forma, utilizou-se o método de análise de componentes principais (PCA).

PCA é um método quantitativamente rigoroso para alcançar a redução desejada, haja vista que através dele é possível gerar um novo conjunto de variáveis, denominados componentes principais [5]. Como cada componente criado é uma combinação das variáveis originais, não há informações redundantes e assim é atingida a simplificação esperada.

\subsection{Extração de características}

Considerando $X$ é um vetor de variáveis aleatórias, as estatísticas utilizadas no presente trabalho são mediana, assimetria e curtose, apresentados nas equações (3), (4) e (5) respectivamente.

$$
\begin{gathered}
M=\frac{X_{(n+1)}}{2} \\
\lambda_{X}=E\left[(X-E(X)) \sigma^{-1}\right]^{3} \\
k_{X}=E\left[(X-E(X)) \sigma^{-1}\right]^{4}
\end{gathered}
$$

\subsection{Métricas de avaliação}

Na etapa de classificação, os valores de acurácia equação (6), sensibilidade equação (7) e especificidade equação (8) foram utilizados como métricas de avaliação.

$$
\begin{gathered}
\text { Acurácia }=\frac{V P+V N}{V P+V N+F P+F N} \times 100 \\
\text { Sensibilidade }=\frac{V P}{V P+F N} \times 100 \\
\text { Especificidade }=\frac{V N}{V N+F P} \times 100
\end{gathered}
$$

Onde, $V P$ representa a quantidade de positivos verdadeiros, $V N$ negativos verdadeiros, FP gravações positivas falsas e $F N$ classificações negativas falsas. 


\subsection{Configurações da máquina de aprendizado}

A máquina de aprendizado utilizada é do tipo supervisionada, onde aplicou-se a rede neural MLP (Multi Layer Perceptron) com duas camadas ocultas. O algoritmo gradiente descendente foi o método utilizado para otimização iterativa, ou seja, treinamento da máquina de aprendizado para atingir valores de erro mínimo. As configurações da rede neural aplicada, seguem na Tabela 1 apresentada abaixo.

Tabela 1: Configurações da Rede Neural MLP.

\begin{tabular}{cc}
\hline Configurações & Valores \\
\hline Taxa de Aprendizado & 0,0001 \\
Número de iterações de treino & 1000 \\
Entradas & 3 \\
Número de neurônios por camada oculta & 100 \\
Número de neurônios na camada de saída & 2 \\
\hline
\end{tabular}

As entradas utilizadas na rede neural foram mediana, curtose e assimetria, presentes nas nas equações (3), (4), (5), enquanto a quantidade de saídas são duas (SAOS ou Saudável).

\section{Resultados e discussão}

Os batimentos cardíacos de ambos os grupos, indivíduos saudáveis e com SAOS, foram classificados a fim de analisar a amostragem dos dados de cada grupo. A partir da classificação dos batimentos cardíacos, foi realizada análise dos gráficos em duas e três dimensões, plotados a partir dos valores estatísticos de mediana, assimetria e curtose obtidos. A Figura 1 é um gráfico de dispersão de assimetria e curtose, a Figura 2 apresenta a dispersão de curtose e mediana e a Figura 3 é um gráfico de dispersão de assimetria e mediana.

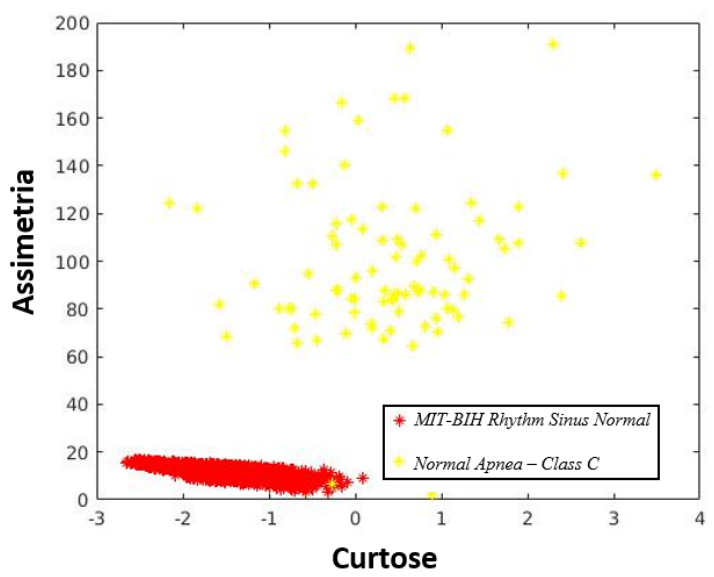

Figura 1: Representação em 2D da dispersão de assimetria e curtose para os sinais dos indivíduos saudáveis e com SAOS em grau leve.

A partir da análise dos gráficos em duas dimensões apresentados nas Figuras 1, 2 e 3 é possível notar uma classificação clara entre indivíduos saudáveis e diagnosticados com SAOS. 


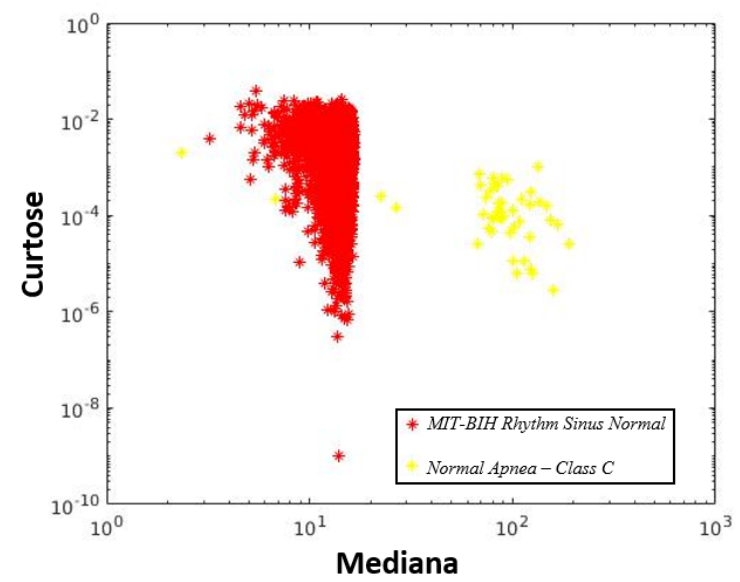

Figura 2: Representação em 2D da dispersão de curtose e mediana para os sinais dos indivíduos saudáveis e com SAOS em grau leve.

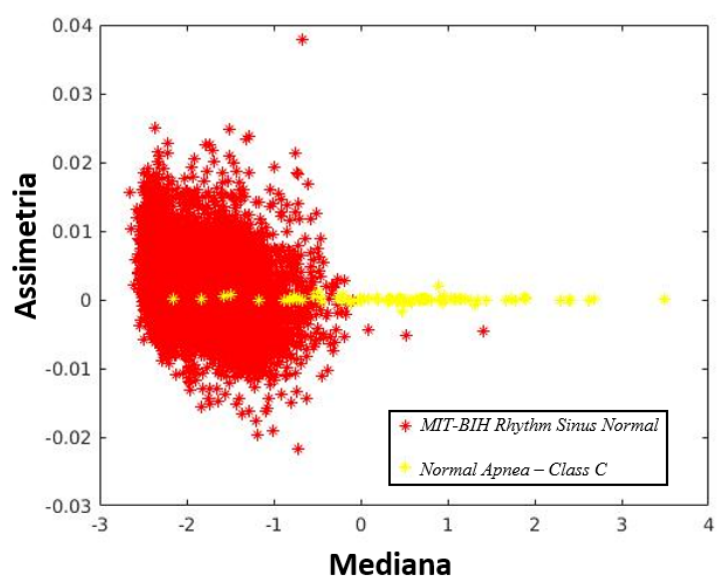

Figura 3: Representação em 2D da dispersão de assimetria e mediana para os sinais dos indivíduos saudáveis e com SAOS em grau leve.

As Figuras 1 e 2 apresentam uma maior separação entre os grupos, reafirmando os estudos em [9], que indicam a curtose como abordagem mais adequada para análise de sinais de ECG.

A Figura 4 apresenta o gráfico em três dimensões, utilizando a mediana, assimetria e curtose dos batimentos de cada grupo. A representação tridimensional da Figura 4 proporciona uma melhor visualização da separação entre ambos os grupos e consequentemente melhor classificação de cada indivíduo.

Essa representação se torna ainda mais benéfica por ocasionar diagnóstico de forma visual e simples, uma vez que interpretar os resultados da polissonografia é uma tarefa bastante complexa, sendo necessário especialistas qualificados em diferentes especialidades médicas, assim como no estudo do sono.

A Figura 5 apresenta os melhores valores de métricas de avaliação encontrados. Esse resultado corresponde ao gráfico de duas dimensões (curtose x mediana) apresentado na Figura 2, indicando que esse gráfico apresenta maior separação entre os grupos e consequentemente uma melhor 
performance.

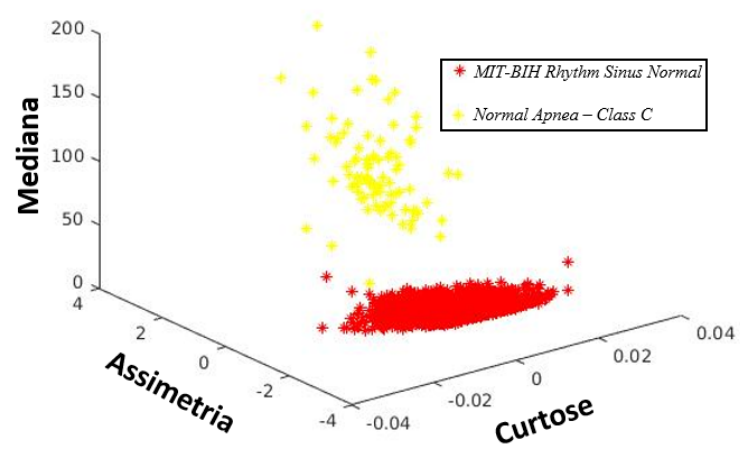

Figura 4: Os sinais avaliados neste estudo, a saber, saudável e SAOS em grau leve, são apresentados em um espaço 3D a partir da mediana, assimetria e curtose de cada grupo.

A partir da Figura 5, nota-se também que apesar do presente artigo trabalhar com SAOS em grau leve, as métricas utilizadas, acurácia, sensibilidade e especificidade, possuem porcentagem igual ou maior que $88 \%$, indicando que o método utilizado ocasiona diagnóstico assertivo mesmo para indivíduos com baixo índice IAH.

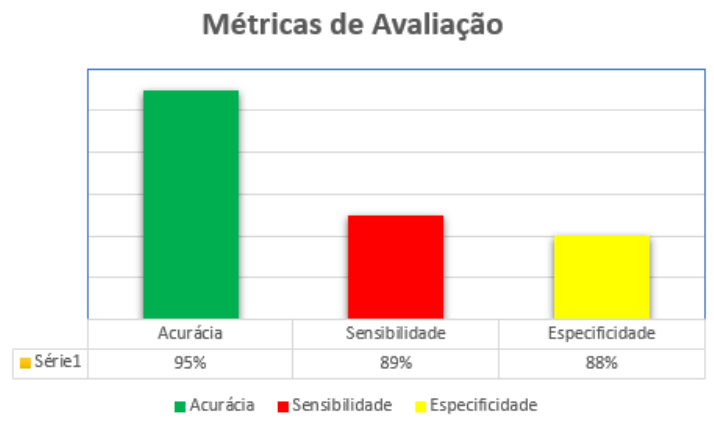

Figura 5: Valores de classificação, em porcentagem, obtidos a partir das métricas de avaliação utilizadas neste estudo, a saber, acurácia, sensibilidade e especificidade.

\section{Conclusão}

Neste artigo, o uso de aprendizado de máquinas e estatísticas de alta ordem possibilitaram a extração de características e classificação de diferentes grupos de indivíduos. Portanto, os resultados obtidos a partir de sinais de ECG, um dos exames do estudo polissonográfico, podem ser utilizados para um diagnóstico de indivíduos com SAOS, auxiliando assim a equipe médica na tomada de decisões de forma mais prática e autônoma. Quando combinado com a evolução tecnológica, esse método apresenta uma simplicidade de diagnóstico ainda maior, visto que com o desenvolvimento de dispositivos que monitoram sinais biológicos cada vez menores, com maior robustez e excelente capacidade de processamento, é possível ocasionar melhora na área da saúde bem como no bem estar do corpo humano. 


\section{Referências}

[1] Bittencourt L. R., Togeiro S. M. and Bagnato M. Diagnóstico da síndrome da apnéia e hipopnéia obstrutiva do sono. Moreira JR Editora; 2012, 19(6): 584-593.

[2] Goldberger A. L., Amaral L. A. N., Glass L., Hausdorff J. M., Ivanov P. C., Mark R. G., Mietus J. E., Moody G. B., Peng C. K. and Stanley H. E. PhysioBank, PhysioToolkit e PhysioNet: componentes de um novo recurso de pesquisa para sinais fisiológicos complexos. Circulation 101(23):e215-e220; 2000 (13 de junho).

[3] Haggsträm F., Zettler E. and FAM C. Apnéia obstrutiva do sono e alterações cardiovasculares. Scientia Medica, Porto Alegre, v. 19, n. 3, p. 122-128, jul./set. 2009.

[4] Ito F. A., et al. Condutas terapêuticas para tratamento da Síndrome da Apnéia e Hipopnéia Obstrutiva do Sono (SAHOS) e da Síndrome da Resistência das vias Aéreas Superiores (SR$v A S)$ com enfoque no Aparelho Anti-Ronco (AAR-ITO). R Dental Press Ortodon Ortop Facial, Maringá, v. 10, n. 4, p.143-156, jul. 2005.

[5] MATHWORKS. Principal Component Analysis (PCA), Help Center (2021). Acesso em: 21/04/2021, 10:16h. Disponível em: https://www.mathworks.com/help/stats/principalcomponent-analysis-pca.html.

[6] Pacheco F., Anjos E. and Maia A. Síndrome da apnéia/hipopnéia obstrutiva do sono: Artigo de revisão bibliográfica. Revista UNILUS Ensino e Pesquisa, v. 12, n. 29, out./dez. 2015 ISSN 2318-2083 (Eletrônico).

[7] Penzel T., Moody G. B., Mark R. G., Goldberger A. L. and Peter J. H. The Apnea - ECG Database. Computers in Cardiology. 2000;27: 255-258.

[8] Pereira A. Síndrome da Apnéia Obstrutiva do Sono: Fisiopatologia, Epidemiologia, Consequências,Diagnóstico e Tratamento. Arquimed, Porto, v. 21, n. 5/6, p.159-173, 2007.

[9] Queiroz J. A., et al. Diagnostic decision support systems for atrial fibrillation based on a novel electrocardiogram approach. Journal of Electrocardiology (2017). Avaidable on. Acesso em 17 de Junho 2020.

[10] SBPT - Sociedade Brasileira de Pneumologia e Tisiologia. Apnéia do Sono. Acesso em: 29/01/2021, 10:48h. Disponível em: https://sbpt.org.br/portal/espaco-saude-respiratoriaapneia-do-sono.

[11] Sher A. E., Schechtman K. B. and Piccirillo J. F. The Efficacy of Surgical Modifications of the Upper Airway in Adults with Obstructive Sleep Apnea Syndrome. Sleep 1996, 19(2): 156-177.

[12] Silva L., Queiroz J. and Barros A. Support method for the diagnosis of Atrial Fibrillation using Machine Learning. XLI CILAMCE, 16 - 19 November, 2020.

[13] Wiegand L. and Zwilich C. W. Obstructive Sleep Apnea. In Bone RC (Ed) Disease-a-Month. St. Louis, Mosby Year Book; 1994, volume XL, 4, pp 199-252. 